\title{
Modelo de balance de línea para mejorar la productividad en una empresa de procesamiento de vidrio templado
}

OMAR ENRIQue Escalante TORRES ${ }^{1}$

Recibido: 03/03/2021 Aceptado: 25/05/2021 Publicado: 26/07/2021

\begin{abstract}
RESUMEN
En el presente trabajo se desarrolló un modelo de balance de línea para una empresa de procesamiento de vidrio templado, con el propósito de demostrar cómo su implementación ayuda a mejorar la productividad. El modelo está basado en el desarrollo de los 5 pasos de la teoría de restricciones (TOC): identificar la restricción, explotar la restricción, subordinar todo a la restricción, elevar la restricción y verificar si existe una nueva restricción. Una vez identificada la restricción, se procedió a explotarla con la aplicación de acciones de mejora, tales como la detección y eliminación de mudas, y con la aplicación de la metodología 5 S hasta lograr una capacidad de producción acorde con la demanda. Dado que fue necesario invertir económicamente, se elaboró un cuadro con una estructura de costos del antes y después del desarrollo del modelo a fin de calcular el valor del ROI para determinar si la inversión realizada favoreció el aumento de la productividad.
\end{abstract}

Palabras clave: balance de línea; productividad; mudas; 5 S; teoría de restricciones.

\section{INTRODUCCIÓN}

En el país, la mayoría de las empresas u organizaciones aplican diversas teorías, técnicas y herramientas de mejora con la finalidad de aumentar su productividad; en muchos casos, se trata de imitar experiencias de mejoras que fueron aplicadas en otras empresas que dieron como resultado un incremento significativo de la productividad y, en consecuencia, una ventaja competitiva en relación a sus competidores. Es fundamental ejecutar de manera correcta un conjunto de herramientas de mejora continua que propicie un verdadero cambio dentro de la organización para mejorar la productividad, reducir costos y obtener un mayor margen de ganancias sostenible en el tiempo.

Las pymes, que representan la mayor parte de las empresas en el país, no cuentan con líneas de producción totalmente automatizadas, por lo que necesitan equilibrar correctamente cada estación de trabajo para así lograr aprovechar al máximo sus recursos. Por lo general, el montaje de maquinarias y la distribución de la línea de producción se realizan de manera empírica, sin aplicar técnicas o herramientas de mejora que ayuden a aprovechar los recursos disponibles en favor del incremento de la productividad. Para estos casos se debería buscar un conjunto de herramientas de mejora continua que se ajuste a las necesidades económicas, de espacio y demanda de cada organización.

El presente estudio se desarrolló en una empresa dedicada al procesamiento de vidrio templado utilizado en el rubro arquitectónico como parte de los acabados de la construcción. La mayoría de las máquinas utilizadas para el procesamiento de vidrio templado son de procedencia italiana y, a menudo, son adquiridas de diferentes proveedores, por motivos económicos o porque el proveedor no ofrece maquinaria para armar toda la línea de procesamiento. Esto hace que existan distintas capacidades de producción en las áreas de trabajo al momento del montaje de la línea de producción.

1 Ingeniero industrial por la Universidad Nacional de Ingeniería. Jefe de planeamiento y control de la producción de Glasstempcorr S.A.C. (Callao, Perú).

ORCID: https://orcid.org/0000-0003-3695-5880

Autor de correspondencia: oescalante@glasstempcorr.com.pe 
El propósito de este artículo es implementar un modelo de balance de línea que comprenderá el uso de herramientas de mejora continua y de lean manufacturing para toda la línea de producción (áreas de corte, pulido, entalle y horno), debido a que la empresa presenta problemas de baja productividad y desaprovechamiento de horas hombre, al no tener las áreas de trabajo correctamente balanceadas en sus capacidades de producción. A diferencia de otros trabajos de investigación y tesis en donde se desarrolla el uso del balance de línea como una herramienta de mejora para el incremento de la productividad basado en la elaboración de estrategias para equilibrar las capacidades de procesamiento de cada área de trabajo, aquí se empleará un "modelo de balance de línea" que consiste en una selección de herramientas de mejora continua y lean manufacturing que permitirá lograr un aumento de la productividad.

El modelo que se utilizará para balancear la línea de procesamiento de vidrio templado se basa en la ejecución de los 5 pasos de la teoría de restricciones (identificar, explotar, subordinar, elevar y repetir). Se utilizarán también herramientas de lean manufacturing (detección y eliminación de mudas) y de mejora continua (metodología 5S) al momento de ejecutar las acciones de mejora del paso 2 (explotar la restricción). Alcalde (2010) menciona que la metodología de las $5 \mathrm{~S}$ se basa en el orden y la limpieza, pues esto permite crear una nueva cultura dentro de la organización. Al utilizar herramientas de mejora continua ya existentes en esta investigación, se podría decir que se está expandiendo su aplicación en el desarrollo de este modelo de balance de línea.

Para implementar el modelo de balance de línea, se tiene que realizar un análisis de los trabajos que se realizan en todas las áreas, esto incluye la revisión de los reportes de producción y los horarios de los turnos para obtener el tiempo que el operario utiliza para la producción en condiciones normales, a una velocidad y ritmo de trabajo que debería estar acorde con la demanda. Con esta información se puede obtener la capacidad de producción de cada área de trabajo en condiciones normales y así determinar cuál tiene la menor capacidad y sería la limitante dentro de la línea de producción.

Por todo lo expuesto, esta investigación tiene como objetivo principal definir si se encuentra una relación entre la implementación de este modelo de balance de línea y una mejora o incremento de la productividad en la empresa de procesamiento de vidrio templado que se está analizando. Será necesario el planteamiento de una hipótesis que posteriormente será contrastada con los resultados para verificar si existe una relación entre el modelo desarrollado y el incremento de la productividad. La hipótesis es la siguiente: "Mediante la aplicación de un modelo de balance de línea, se mejorará la productividad de una empresa de procesamiento de vidrio templado".

Asimismo, se elaborará una estructura de costos al inicio y al final de la aplicación del modelo para analizar el impacto económico del precio de venta del producto terminado sobre la empresa y, finalmente, se calculará el ROI para determinar si la inversión realizada fue beneficiosa para la empresa.

\section{Balance de línea}

Para Salazar (2019), el balance de línea es un método de gran importancia para controlar la producción, debido a que, si se logra un correcto equilibrio de la línea de producción, se podrá optimizar otras variables que influyen en la productividad, por ejemplo: el inventario de piezas por procesar, el tiempo promedio de procesamiento y los despachos parciales de piezas terminadas.

Según los autores Suñé et al. (2004), al momento de diseñar la línea de producción, se deben distribuir las tareas de tal manera que los recursos sean aprovechados en todo el proceso. Lo más complicado de balancear la línea de producción es dividir el proceso en estaciones de trabajo donde se ejecutará un grupo de tareas, de manera que la carga de trabajo sea la más óptima dentro del tiempo de ciclo de la producción. A propósito de esto último, García et al. (2004) indican que el objetivo de balancear una línea de producción es que cada estación de trabajo produzca un mismo metraje y tenga un mismo tiempo de ciclo de producción, para así evitar las acumulaciones de carga por procesar.

Los pasos para iniciar el balance de línea para Suñé et al. (2004) son los siguientes:

1. Definir e identificar todas las tareas que conforman al proceso de producción.

2. Determinar el tiempo estándar que se necesitará para el desarrollo de cada tarea.

3. Establecer los recursos que se necesitarán.

4. Determinar el orden de ejecución.

Meyers (2000) sostiene que una vez implementado el balance de línea se pueden identificar los cuellos de botella. La variabilidad de la demanda y el poseer maquinaria con capacidades diferentes hace 
que se requieran diferentes tiempos de procesamiento, lo que genera cuellos de botella dinámicos.

Para obtener una línea de producción balanceada, se necesita de una correcta aplicación teórica, redistribución de recursos e incluso inversiones económicas.

Para Calampa (2014), al balancear la línea de producción se logra:

- Reducir y estandarizar los costos.

- Elaborar una estructura de bonificaciones por la productividad alcanzada.

- Obtener la cantidad de producción esperada dentro del plazo establecido.

- Mejorar la gestión de la producción.

- Incrementar la productividad general y el estímulo del personal.

- Determinar tiempos y movimientos para mejorar la distribución de la carga de trabajo.

- Eliminar los inventarios de piezas en proceso y los cuellos de botella.

- Mejorar el flujo del proceso entre las áreas de producción.

- Comprometer a los trabajadores en la eliminación de los 7 desperdicios o mudas del lean Manufacturing (sobreproducción, tiempos de espera, transporte, procesos, inventarios, movimientos y productos defectuosos).

\section{Teoría de restricciones (TOC)}

En el balance de línea encontramos el término "cuello de botella". En la Teoría de restricciones (TOC, por sus siglas en inglés) de Goldratt (2004), se menciona el concepto de "cuello de botella" como la falta de capacidad de una estación de trabajo para satisfacer la demanda de una estación anterior, estableciendo de esta manera una "restricción". Según Saidi et al. (2017), son estos "cuellos de botella" los que determinan las capacidades de producción. En consecuencia, es la estación de trabajo con la mínima capacidad entre todas la que está generando un impacto negativo en el tiempo de ciclo total. Para Kikolski (2016), esto marca el ritmo del proceso productivo.

Chase et al. (2009) indican que la teoría de restricciones representa un conjunto de herramientas de mejora continua, la cual se logra aplicando estos 5 pasos:
1. Identificar la restricción. Se considera un paso complicado, pues es necesario evaluar el proceso en su totalidad y así detectar aquella actividad que restringe el flujo del proceso. Esto no se refiere solo al proceso, sino también a toda la organización.

2. Explotar la restricción. Se requiere encontrar métodos que sean capaces de aumentar la capacidad de la actividad limitante, es decir aumentar el throughput (rapidez del sistema para aumentar sus ganancias).

3. Subordinar todo a la restricción. Se hace que la limitante opere al $100 \%$ de su capacidad máxima, puesto que es la que marca el ritmo de trabajo.

4. Elevar la restricción. Se debe aumentar la capacidad de la restricción, aquí se puede considerar el uso de recursos económicos.

5. Verificar si existe una nueva restricción. Si la limitante inicial es eliminada, se procede a reiniciar los pasos anteriores con la siguiente limitante del proceso productivo.

\section{Metodología de las 5S}

Según Hernández (2016), el objetivo de las 5 S es mejorar el orden de las áreas de trabajo y que esto se mantenga en el tiempo; de este modo, será posible perfeccionar las condiciones de trabajo, el clima organizacional y el desempeño laboral, lo que resultará en el aumento de la productividad y la mejora de la calidad de los productos.

La primera $\mathrm{S}$, seiri (clasificación), según Bonilla et al. (2010), consiste en clasificar los objetos en útiles y no útiles para solamente conservar los que son de utilidad y retirar los que no lo son porque ocupan espacio.

La segunda $\mathrm{S}$, seiton (ordenar), según Bonilla et al. (2010), consiste en organizar los elementos clasificados del paso anterior, de manera que se puedan ubicar con facilidad, lo que permite ahorrar tiempo y evitar movimientos innecesarios.

La tercera S, seiso (limpieza), consiste en identificar y eliminar las fuentes de suciedad; según lo mencionado por Rey (2005), se debe realizar el aseo de la zona de trabajo para mantenerla libre de suciedad e incentivar al trabajador a que lo realice de forma diaria al inicio y final de la jornada laboral.

La cuarta S, seiketsu (estandarización), parte de las $3 \mathrm{~S}$ anteriores y su finalidad es evitar el retraso en 
las etapas previas, cuya ejecución debe ser parte de la rutina diaria. Para Cuatrecasas y Torrel (2010), la estandarización trata de formar un hábito en los trabajadores para el cumplimiento de los pasos anteriores. Tener una buena organización y orden será esencial para lograr la estandarización.

La última $\mathrm{S}$, shitsuke (disciplina), según Dorbessan (2010), es la parte más trascendente debido a que se encarga de buscar el cumplimiento de las $4 \mathrm{~S}$ anteriores. Impulsa la práctica de la disciplina con un espíritu proactivo, ya que los beneficios serán provechosos, tanto para la empresa como para los trabajadores. En la medida que los trabajadores sean disciplinados y comprometidos, la empresa logrará grandes resultados en cuanto a calidad y niveles de productividad.

\section{Mudas: Desperdicios}

Uno de los objetivos básicos del lean manufacturing es eliminar o disminuir los desperdicios o mudas que resultan de diversas tareas y que no generan valor alguno. Socconini (2019) menciona que el lean manufacturing clasifica las variedades de mudas en siete grupos:

- Sobreproducción: consiste en producir en exceso a lo programado.

- Espera: tiempo de retraso que se genera entre el operador y la máquina que está operando.

- Transporte de materiales y herramientas: desplazamiento inútil de personas o piezas en proceso que no genera valor.

- Procesos innecesarios: procesos que no incrementan el valor del producto final.

- Sobreinventario: materiales, piezas en proceso o productos terminados que se producen en abundancia y exceden la demanda del área comercial.

- Movimientos innecesarios del trabajador: movimientos de materiales o herramientas que se realizan dentro del proceso, que no generan valor y, por el contrario, incrementan los costos.

- Productos defectuosos: pérdida de recursos que se utilizaron al momento de producir un producto no conforme.

\section{Productividad}

Para García (2006), la productividad es el nivel de rendimiento con que se utilizan los recursos disponibles para lograr los objetivos; indica también que la productividad de una empresa se examina con el fin de determinar los problemas que la afectan $y$, posteriormente, establecer las estrategias para darles solución.

La OIT (1996) define la productividad como una relación entre las unidades producidas y el insumo utilizado, indica además que la productividad puede verse afectada por diferentes factores externos, así como por deficiencias en sus actividades internas.

\section{METODOLOGÍA}

La presente investigación es de tipo aplicada y de diseño experimental, dado que la variable independiente "modelo de balance de línea" se manipula de manera intencional, lo cual produce un efecto en la variable dependiente "productividad".

\section{Línea de procesamiento de vidrio templado}

El estudio se realizó en la empresa Glasstempcorr, ubicada en el distrito de Ventanilla. La planta está sobre un terreno de aproximadamente $5000 \mathrm{~m}^{2}$ y la línea de procesamiento está compuesta por las áreas de corte, canteo, entalle, lavadora y horno. En la actualidad, la productividad de la línea de procesamiento no se encuentra en un nivel adecuado debido al poco control de los trabajos entre las áreas, los reprocesos y desechos. Por esa razón, se recurre a la aplicación de un modelo de balance de línea basado en el desarrollo de los 5 pasos de la teoría de restricciones, como herramienta de mejora continua, para que al momento de aplicar las acciones de mejora se utilicen la detección y eliminación de mudas de manera conjunta con la implementación de la metodología de las $5 S$ en el área de trabajo en donde se detecte la restricción. Este modelado se puede apreciar de manera gráfica en la Figura 1.

\section{Evaluación e implementación de la teoría de restricciones (TOC)}

Se procede con la aplicación del paso 1. Como se cuenta con la data histórica de producción, se pueden obtener las capacidades de cada área y un promedio de metros cuadrados de vidrio templado procesado de los últimos 3 meses. Con esos datos es posible determinar el área que tiene la menor capacidad de procesamiento y por lo tanto genera la restricción. En la Figura 2, se concluye que la restricción de la línea recae en el área de entalle. En esta etapa inicial, se puede elaborar una estructura de costos para hallar el valor del costo unitario y así compararlo con el valor que se obtendría después de la implementación del modelo de balance de línea. 


\begin{tabular}{|c|c|c|c|c|c|c|c|}
\hline Inicio & Paso 1 & \multicolumn{2}{|c|}{ Paso 2} & Paso 3 & Paso 4 & Paso 5 & Resultados \\
\hline \begin{tabular}{|l|} 
- Revisión de la \\
data histórica \\
de producción \\
(últimos 3 \\
meses). \\
- Cálculo de la \\
capacidad de \\
producción \\
diaria de cada \\
área de trabajo.
\end{tabular} & $\begin{array}{l}\text { Se identifica la } \\
\text { restricción. } \\
\text { - Con la } \\
\text { información } \\
\text { anterior se } \\
\text { determina cual } \\
\text { es el área de } \\
\text { procesamiento } \\
\text { que tiene la } \\
\text { restricción. }\end{array}$ & \begin{tabular}{|c} 
Se explota \\
- Priorizar \\
recursos \\
aplican 2 he \\
mejora \\
\end{tabular} & \begin{tabular}{l} 
restricción. \\
el uso de \\
opios, se \\
amientas de \\
ntinua. \\
\multicolumn{1}{|c|}{} \\
$\begin{array}{l}\text { Detección y } \\
\text { eliminación } \\
\text { de mudas. }\end{array}$ \\
\end{tabular} & $\begin{array}{l}\text { Se subordina } \\
\text { los recursos a la } \\
\text { restricción } \\
\text { anterior. } \\
\text { - El proceso que } \\
\text { tenga la } \\
\text { restricción es el } \\
\text { que marque el } \\
\text { ritmo de } \\
\text { trabajo. }\end{array}$ & $\begin{array}{l}\text { Se eleva la } \\
\text { restricción. } \\
\text { - Aquí se puede } \\
\text { hacer uso de } \\
\text { una inversión } \\
\text { economica para } \\
\text { aumentar la } \\
\text { restricción si es } \\
\text { necesario. }\end{array}$ & $\begin{array}{l}\text { Se verifica si } \\
\text { hay una nueva } \\
\text { restricción } \\
\text { - Si la } \\
\text { restricción } \\
\text { inicial es } \\
\text { eliminada se } \\
\text { realizan los } \\
\text { pasos } \\
\text { anteriores. }\end{array}$ & $\begin{array}{l}\text { Prueba de } \\
\text { hipótesis, se } \\
\text { realiza la } \\
\text { comprobación. } \\
\text { Análisis } \\
\text { económico, se } \\
\text { comparan los } \\
\text { costos unitarios } \\
\text { (inicial - final) }\end{array}$ \\
\hline
\end{tabular}

Figura 1. Secuencia del modelo de balance de línea.

Fuente: Elaboración propia.

\begin{tabular}{|c|c|c|c|c|c|}
\hline INICIAL & $\begin{array}{c}\text { Paso } 1 \\
\text { Corte }\end{array}$ & $\begin{array}{c}\text { Paso } 2 \\
\text { Canteo }\end{array}$ & $\begin{array}{c}\text { Paso } 3 \\
\text { Entalle }\end{array}$ & $\begin{array}{c}\text { Paso } 4 \\
\text { Lavado }\end{array}$ & $\begin{array}{l}\text { Paso } 5 \\
\text { Templado }\end{array}$ \\
\hline MAQUINARIA & Cortadora & Canteadora & Taladro & Lavadora & $\begin{array}{l}\text { Horno de } \\
\text { templado }\end{array}$ \\
\hline $\begin{array}{l}\text { CAPACIDAD DE } \\
\text { PRODUCCIÓN } \\
\text { DIARIA (M2) }\end{array}$ & 1450 & 1200 & 800 & 1500 & 1250 \\
\hline
\end{tabular}

Figura 2. Capacidades iniciales de producción en la línea de procesamiento.

Fuente: Elaboración propia.

Para el paso 2, se requiere explotar la restricción, por lo que se tienen que realizar acciones que ayuden a incrementar la capacidad de procesamiento del área de entalle solo con los recursos propios del área (operarios y maquinaria). Se aplican dos herramientas de mejora continua de forma paralela, las cuales forman parte del modelo propuesto; la primera fue la implementación de las 5S. Previo a dicha implementación, se realizó una línea base, se tomaron fotos de las condiciones anormales y se otorgó un puntaje a cada categoría (clasificación, orden, limpieza, estandarizar y mantener) para así poder determinar los puntos a corregir. El jefe de calidad, con el apoyo de los encargados del área, dirige la implementación, la cual constaba de tres fases: en la primera fase, se capacita y sensibiliza al personal involucrado; en la segunda fase, se implementa la metodología; y en la tercera fase, se realiza un seguimiento de todo lo realizado anteriormente.

La segunda herramienta es la detección y eliminación de las mudas que existan dentro del proceso productivo, la cual es utilizada en lean manufacturing. Al analizar el proceso de entalle, se encuentran mudas relacionadas con tiempos de espera ocasionadas por falta de información de los trabajos a realizar y por paradas debido a mantenimientos no programados; asimismo, se detecta un porcentaje considerable de productos defectuosos, lo cual genera desperdicio de materia prima y horas-hombre. Se tienen que desarrollar planes de acción para reducir estos desperdicios, tales como preparar con anticipación toda la información de los trabajos a realizar, mejorar el programa mensual de mantenimiento preventivo y capacitar al personal para disminuir los productos defectuosos por mala manipulación. Una vez ejecutadas las acciones de mejora, la línea de procesamiento quedaría balanceada, tal como se puede apreciar en la Figura 3.

El paso 3 consiste en subordinar todo lo demás a la actual capacidad de la restricción, lo que significa que el área de entalle es la que marca el ritmo de trabajo y, por consiguiente, se deben racionar los recursos que se emplean en las demás áreas de producción (operario y maquinaria) hasta lograr una capacidad que supere por poco a la de entalle para así lograr una capacidad de procesamiento homogénea en toda la línea. Es pertinente contar con el apoyo de la gerencia para llevar a cabo este paso, puesto que con frecuencia se debe convencer a los directivos 
sobre la necesidad de reducir el ritmo de trabajo en otras áreas que generan producción en exceso.

El paso 4 consiste en elevar la restricción, para lo cual se necesitará hacer una inversión económica que consiste en la adquisición de un taladro manual y la contratación de 2 operarios, con lo cual la capacidad del área de entalle incrementa a 1300 $\mathrm{m}^{2}$, como se puede apreciar en la Figura 4. En esta etapa se puede elaborar otra estructura de costo unitario para compararlo con el inicial.

El paso 5 consiste en verificar si el proceso limitante quedó eliminado. En nuestro caso se puede apreciar que ahora el área de entalle dejó de ser una restricción y el área de canteo pasó a serlo; por lo cual, se tendrían que ejecutar nuevamente los pasos anteriores hasta obtener una capacidad de procesamiento acorde a la demanda planteada por el área comercial.

\section{RESULTADOS}

La aplicación del modelo de balance de línea permite comparar la capacidad de producción antes y después de la implementación del modelo. En esta investigación, se realizará primero una contrastación de la hipótesis planteada, la cual consiste en determinar si se logró incrementar la productividad de la línea de procesamiento de vidrio templado. Para esto, se presenta la Tabla 1 con información de la producción extraída desde enero de 2019 hasta febrero del 2020, en donde se puede apreciar el índice de productividad de los meses en los que se aplicó el modelo de balance

\begin{tabular}{|c|c|c|c|c|c|}
\hline INICIAL & $\begin{array}{c}\text { Paso } 1 \\
\text { Corte }\end{array}$ & $\begin{array}{c}\text { Paso } 2 \\
\text { Canteo }\end{array}$ & $\begin{array}{c}\text { Paso } 3 \\
\text { Entalle }\end{array}$ & $\begin{array}{c}\text { Paso } 4 \\
\text { Lavado }\end{array}$ & $\begin{array}{c}\text { Paso } 5 \\
\text { Templado }\end{array}$ \\
\hline MAQUINARIA & Cortadora & Canteadora & Taladro & Lavadora & $\begin{array}{l}\text { Horno de } \\
\text { templado }\end{array}$ \\
\hline $\begin{array}{l}\text { CAPACIDAD DE } \\
\text { PRODUCCIÓN } \\
\text { DIARIA (M2) }\end{array}$ & 1450 & 1200 & 800 & 1500 & 1250 \\
\hline MAQUINARIA & Cortadora & Canteadora & Taladro & Lavadora & $\begin{array}{l}\text { Horno de } \\
\text { templado }\end{array}$ \\
\hline $\begin{array}{l}\text { CAPACIDAD DE } \\
\text { PRODUCCIÓN } \\
\text { DIARIA (M2) }\end{array}$ & 1450 & 1200 & 1000 & 1500 & 1250 \\
\hline
\end{tabular}

Figura 3. Valores de capacidades de producción luego de la aplicación de acciones de mejora del paso 2.

Fuente: Elaboración propia.

\begin{tabular}{|c|c|c|c|c|c|c|}
\cline { 3 - 6 } \multicolumn{1}{|c|}{$\begin{array}{c}\text { Paso 1 } \\
\text { FINAL }\end{array}$} & \multirow{2}{*}{$\begin{array}{c}\text { Paso 2 } \\
\text { Canteo }\end{array}$} & $\begin{array}{l}\text { Paso 3 } \\
\text { Entalle }\end{array}$ & $\begin{array}{l}\text { Paso 4 } \\
\text { Lavado }\end{array}$ & $\begin{array}{c}\text { Paso 5 } \\
\text { Templado }\end{array}$ \\
\hline MAQUINARIA & Cortadora & Canteadora & Taladro & Lavadora & $\begin{array}{c}\text { Horno de } \\
\text { templado }\end{array}$ \\
\hline $\begin{array}{c}\text { CAPACIDAD DE } \\
\text { PRODUCCIÓN } \\
\text { DIARIA (M2) }\end{array}$ & 1450 & 1200 & 1300 & 1500 & 1250 \\
\hline
\end{tabular}

Figura 4. Valores de capacidades al final del paso 4.

Fuente: Elaboración propia. 
línea. En la información presentada del año 2019, se puede apreciar el índice de productividad antes de la aplicación del modelo de balance de línea. De enero a junio de 2020, se aprecia un incremento del índice de productividad debido a las primeras acciones de mejora realizadas con los propios recursos del área; es importante tener en cuenta que en marzo solo se trabajó hasta el día 16 y en abril no se registra la cantidad de producción puesto que la planta estuvo cerrada por la pandemia del COVID-19. Finalmente, entre julio de 2020 y febrero de 2021 se obtienen otros valores del índice de productividad después de la aplicación del paso 4 de la teoría de restricciones (TOC).

\section{Prueba de hipótesis}

Para comprobar la hipótesis planteada, primero se extrae información de la productividad de la Tabla 1 y se ejecuta en el programa Minitab 18 para así poder determinar si la data tiene o no distribución normal.

Del Minitab 18, se extraen las figuras 5 y 6 del comparativo de la productividad al inicio y después de la aplicación el modelo.

De la prueba de normalidad realizada, se determinó que la distribución es normal debido a que el $p$-valor es mayor que 0.05 , la curva que se muestra tiene forma de campana y, si se traza una línea vertical, cruza la media y la mediana.

Una vez determinado que, para la información recolectada, la data tiene un comportamiento paramétrico, se procede a utilizar la prueba t de Student para 2 muestras para comprar la productividad al inicio y al final de la aplicación del modelo y demostrar la hipótesis.

Tabla 1. Estructura de costos inicial para el paso 1.

\begin{tabular}{|c|c|c|c|c|}
\hline Año & Mes & Metraje $\left(m^{2}\right)$ & Horas empleadas & Índice de productividad (\%) \\
\hline \multirow{12}{*}{2019} & Enero & 765 & 7.01 & 0.84 \\
\hline & Febrero & 775 & 7.05 & 0.85 \\
\hline & Marzo & 785 & 7.15 & 0.88 \\
\hline & Abril & 776 & 6.98 & 0.85 \\
\hline & Mayo & 762 & 6.85 & 0.82 \\
\hline & Junio & 789 & 6.89 & 0.85 \\
\hline & Julio & 782 & 7.08 & 0.87 \\
\hline & Agosto & 780 & 7.11 & 0.87 \\
\hline & Setiembre & 792 & 7.07 & 0.87 \\
\hline & Octubre & 791 & 7.18 & 0.89 \\
\hline & Noviembre & 788 & 7.28 & 0.90 \\
\hline & Diciembre & 793 & 7.25 & 0.90 \\
\hline \multirow{12}{*}{2020} & Enero & 945 & 7.39 & 0.87 \\
\hline & Febrero & 978 & 7.55 & 0.92 \\
\hline & Marzo & 905 & 7.45 & 0.84 \\
\hline & Abril & -- & -- & -- \\
\hline & Mayo & 931 & 7.58 & 0.88 \\
\hline & Junio & 945 & 7.55 & 0.89 \\
\hline & Julio & 1078 & 7.75 & 0.87 \\
\hline & Agosto & 1088 & 7.85 & 0.89 \\
\hline & Setiembre & 1058 & 7.81 & 0.86 \\
\hline & Octubre & 1138 & 7.85 & 0.93 \\
\hline & Noviembre & 1105 & 7.78 & 0.90 \\
\hline & Diciembre & 1158 & 7.89 & 0.95 \\
\hline \multirow{2}{*}{2021} & Enero & 1098 & 7.9 & 0.90 \\
\hline & Febrero & 1115 & 7.68 & 0.89 \\
\hline
\end{tabular}

Fuente: Elaboración propia. 


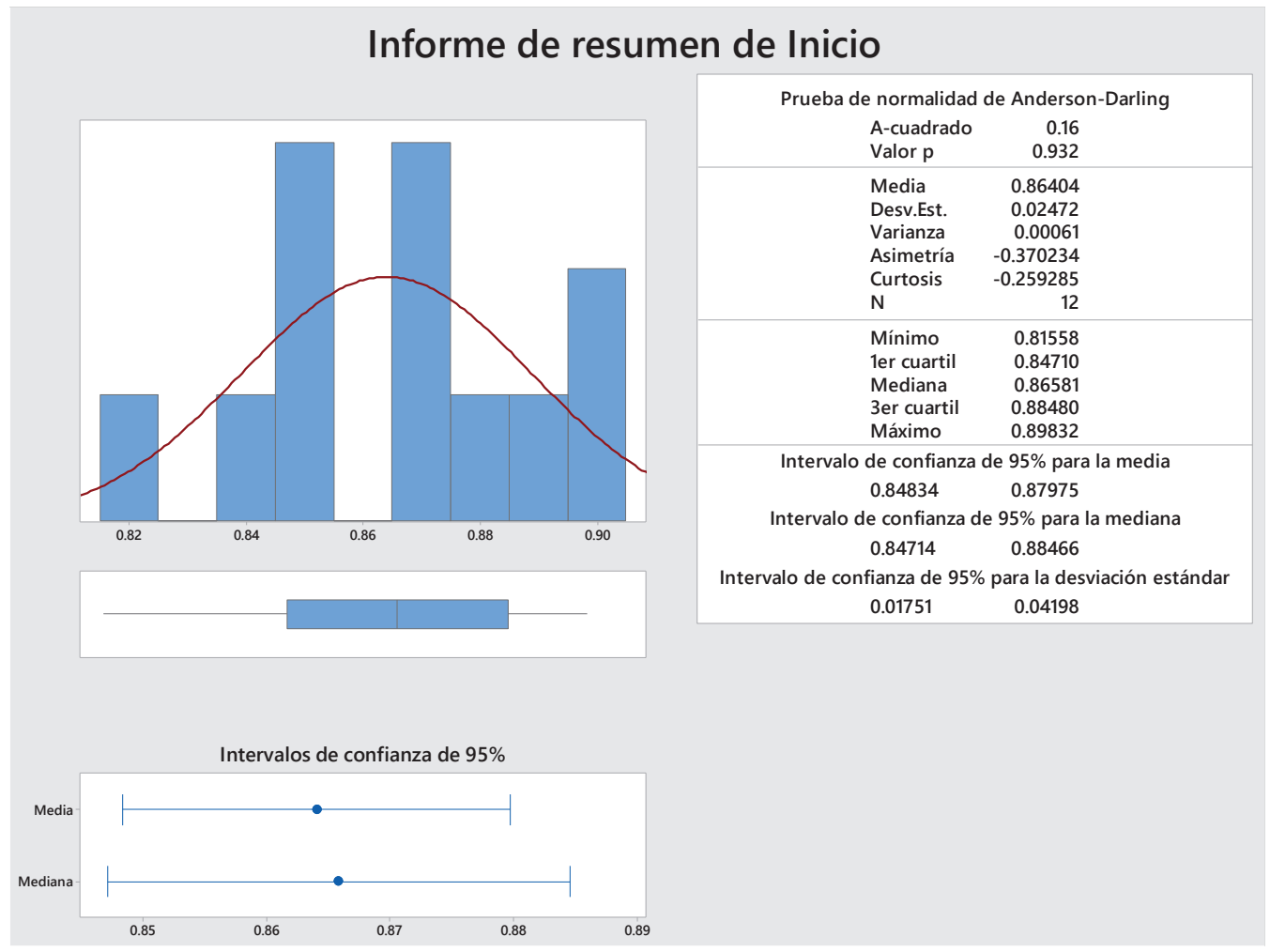

Figura 5. Gráfica de la distribución de la normalidad de los valores de la productividad al inicio de la aplicación.

Fuente: Elaboración propia.

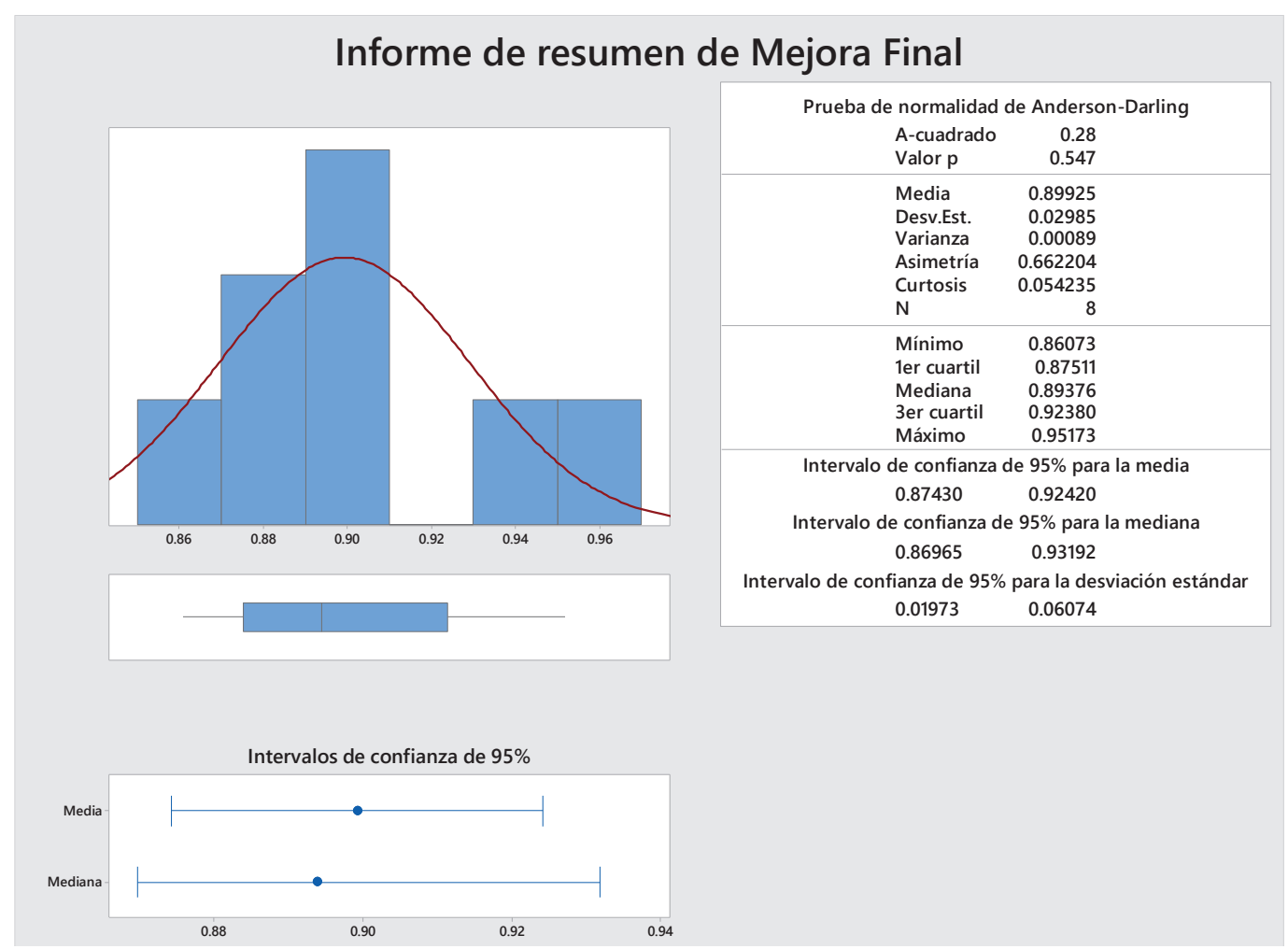

Figura 6. Gráfica de la distribución de la normalidad de los valores de la productividad después de la aplicación del modelo.

Fuente: Elaboración propia. 


\section{Prueba T e IC para dos muestras: Inicio y Mejora final}

\section{Método}

$\mu_{1}$ : media de Inicio

$\mu_{2}:$ media de Mejora final

Diferencia: $\mu_{1}-\mu_{2}$

No se presupuso igualdad de varianzas para este análisis.

\section{Estadísticas descriptivas}

\begin{tabular}{lcccc}
\multicolumn{1}{c}{ Muestra } & N & Media & Desv.Est. & $\begin{array}{c}\text { Error estándar de } \\
\text { la media }\end{array}$ \\
\hline Inicio & 12 & 0.8640 & 0.0247 & 0.0071 \\
Mejora Final & 8 & 0.8993 & 0.0298 & 0.011
\end{tabular}

\section{Estimación de la diferencia}

\begin{tabular}{ll} 
Diferencia & Límite superior de $95 \%$ para la diferencia \\
\hline-0.0352 & -0.0126
\end{tabular}

\section{Prueba}

\begin{tabular}{lll}
$\begin{array}{l}\text { Hipótesis nula } \\
\text { Hipótesis alterna }\end{array}$ & $\begin{array}{l}\mathrm{H}_{0}: \mu_{1}-\mu_{2}=0 \\
\mathrm{H}_{1}: \mu_{1}-\mu_{2}<0\end{array}$ \\
Valor $\mathbf{T}$ & GL & Valor $\boldsymbol{p}$ \\
\hline-2.76 & 13 & 0.008
\end{tabular}

Se tiene en consideración que si se obtiene un $p$-valor $\geq 0.05$, se aceptará como respuesta válida la $\mathrm{H}_{0}$. Pero si se obtiene un $p$-valor $<0.05$, se aceptará como respuesta válida la $\mathrm{H}_{\mathrm{a}}$ y se rechaza la $\mathrm{H}_{0}$.

Dado que el valor de $p$ es menor que 0.05 , se debe rechazar la hipótesis nula y aceptar la alterna, la cual indica que la diferencia $\mu_{1}-\mu_{2}<0$, lo cual demuestra que el porcentaje de productividad aumentó a partir de julio del 2020. Con esta comparación se constata que la hipótesis es correcta a I afirmar que "Mediante la aplicación de un modelo de línea, se mejorará la productividad de una empresa de procesamiento de vidrio templado".

\section{Análisis económico}

Se realizará un comparativo de los costos unitarios promedios al inicio y después de la aplicación de los 5 pasos del TOC para analizar los resultados obtenidos. En la Tabla 2, se puede apreciar una estructura de costos al inicio del estudio.

Del cuadro presentado, se deduce que el valor unitario de venta por $\mathrm{m}^{2}$ es de $\mathrm{S} / 77.34$, que equivale a $\$ 21.48 \approx \$ 22.00$, el precio actual del mercado por $\mathrm{m}^{2}$ para un vidrio de 8 milímetros de espesor, esto calculado para una capacidad de procesamiento de línea de $800 \mathrm{~m}^{2}$. En la Tabla 3, se puede apreciar la estructura de costos resultante después de la aplicación del paso 4.

Del cuadro final, se observa que el valor unitario de venta por $\mathrm{m}^{2}$ ha disminuido a $\mathrm{S} / 69.16$, que equivale a $\$ 19.21 \approx \$ 20.00$, esto también está calculado para vidrios de 8 milímetros de espesor y para una capacidad final de procesamiento de la línea de $1200 \mathrm{~m}^{2}$. En esta tabla, se ha considerado el costo de la maquinaria adquirida (1 taladro) y su valor se ha distribuido para una vida útil de 5 años. El monto del precio de venta final está por debajo del precio del mercado, lo que permite a la empresa ofrecer al cliente un precio menor al del mercado sin afectar sus ganancias.

Se realiza un análisis sobre la rentabilidad de la inversión en el área de entalle para determinar qué

Tabla 2. Estructura de costos inicial para el paso 1.

Estructura de costos - Condiciones iniciales

Valores promedio para una demanda de $800 \mathrm{~m}^{2}$

\begin{tabular}{|c|c|c|c|c|}
\hline Vidrio templado & Costo total & Cantidad $\left(\mathrm{m}^{2}\right)$ & Co & nitario \\
\hline Material (vidrio crudo) & S/ $\quad 39,000.00$ & 800 & $\mathrm{~S} /$ & 48.75 \\
\hline M.O. (60 operarios) & $\mathrm{S} / \quad 3,000.00$ & 800 & $\mathrm{~S} /$ & 3.75 \\
\hline GIF (2.5) & S/ $\quad 7,500.00$ & 800 & $\mathrm{~S} /$ & 9.38 \\
\hline Costo de fabricación & $S / \quad 49,500.00$ & 800 & s/ & 61.88 \\
\hline Precio de venta $(25 \%)$ & S/ $\quad 61,875.00$ & & s/ & 77.34 \\
\hline
\end{tabular}

GIF = Gasto indirecto de fabricación que equivale a 2.5 veces el costo de la mano de obra (M.O.)

Fuente: Elaboración propia. 
tan lucrativa resultó para la empresa. De los valores obtenidos en las Tablas 1 y 2 , se elabora la Tabla 4 para hallar el valor del retorno sobre la inversión (ROI) utilizando la fórmula mostrada abajo.

$$
\begin{aligned}
& R O I=\frac{G A N A N C I A-I N V E R S I O ́ N}{I N V E R S I O ́ N} \\
& \begin{array}{rll}
\mathbf{G} & =\mathrm{S} / 528,000.00 & \text { Ganancia } \\
\mathbf{I} & =\mathrm{S} / 422,350.00 & \text { Inversión }
\end{array}
\end{aligned}
$$

$$
\mathrm{ROI}=0.20
$$

Del resultado obtenido, se puede deducir que por cada sol que se ha invertido en este modelo de balanceo de línea se obtendrán S/ 0.20 de ganancia, lo cual hace rentable al proyecto.

\section{DISCUSIÓN}

Este modelo de balance de línea tiene como estructura principal la ejecución de los 5 pasos de la teoría de restricciones. A partir de esto, al momento de la aplicación del paso 2 , se identificó una serie de acciones de mejora, pero sin la ayuda de inversión económica, utilizando únicamente recursos propios del área analizada en la cual se detectó la restricción. Para el desarrollo de las acciones de mejora, se contempló la eliminación de las mudas, que son los desperdicios detectados en el área de entalle, $y$, de manera conjunta, se implementaron las 5S, con énfasis en las tres primeras, debido a que se obtuvo una calificación baja en el informe de la línea base. Durante la ejecución de la metodología 5S, el jefe de calidad designó como responsables de la ejecución a los encargados del área de producción "entalle" (uno de cada turno: día y noche), los cuales tienen a su cargo la distribución de tareas de 5 operarios. Para Vargas (2002), la designación de un responsable es importante puesto que ayuda con el cumplimiento continuo de esta herramienta y, además, contribuye con la integración y capacitación de los trabajadores.

El modelo de balance de línea (variable independiente) aplicado influyó la productividad (variable dependiente), dado que se validó la hipótesis planteada por medio de un software estadístico. Tras el análisis de la estructura de costos final, se obtuvo un precio unitario de venta de $S / 69.11$, que es menor al precio unitario de venta de la estructura de costos inicial, S/ 77.34. Esto resulta favorable para la empresa porque el producto se puede ofrecer a un precio inferior al del mercado, lo que permite captar más clientes sin que el margen de ganancias

Tabla 3. Estructura de costos al final del paso 4.

Estructura de costos - Condiciones finales

\begin{tabular}{|c|c|c|c|c|c|}
\hline Vidrio templado & & Costo total & Cantidad $\left(\mathrm{m}^{2}\right)$ & \multicolumn{2}{|c|}{ Costo unitario } \\
\hline Material (vidrio crudo) + Maq. & S/ & 55544.00 & 1200 & $\mathrm{~S} /$ & 46.29 \\
\hline M.O. (62 operarios) & $\mathrm{S} /$ & 3100.00 & 1200 & $\mathrm{~S} /$ & 2.58 \\
\hline GIF (2.5) & S/ & 7750.00 & 1200 & S/ & 6.46 \\
\hline Costo de fabricación & S/ & 66394.00 & 1200 & $\mathbf{S} /$ & 55.33 \\
\hline Precio de venta (25\%) & $\mathrm{S} /$ & 82992.00 & & $\mathbf{S} /$ & 69.16 \\
\hline
\end{tabular}

Valores promedio para una demanda de $1200 \mathrm{~m}^{2}$

Se adquirió un taladro manual y se contrató a dos operarios.

GIF = Gasto indirecto de fabricación que equivale a 2.5 veces el costo de la mano de obra (M.O.)

Fuente: Elaboración propia.

Tabla 4. Estructura de producción mensual.

\begin{tabular}{|l|l|c|c|c|c|r|}
\cline { 2 - 5 } \multicolumn{2}{c|}{} & \multicolumn{2}{c|}{ Capacidad de producción $\left(\mathbf{m}^{2}\right)$} & \multirow{2}{*}{ Precio de venta unitario } & Total mensual & Incremento de ventas \\
\cline { 2 - 6 } & Diaria & Mensual & & & S/ 528000.00 \\
\hline $\mathbf{2}$ & Con los recursos actuales & 800 & 20000 & $\mathrm{~S} / 77.34$ & $\mathrm{~S} / 1546800.00$ & $\mathrm{~S}$ \\
\hline
\end{tabular}

Fuente: Elaboración propia. 
se vea afectado. La línea de producción quedará balanceada a una capacidad de $1200 \mathrm{~m}^{2}$, lo cual representa un aumento de $400 \mathrm{~m}^{2}$ sobre la capacidad inicial; este balance de línea permitió aprovechar los recursos del área, tanto el uso y disponibilidad de la maquinaria como el tiempo empleado por los operarios.

Para Niebel y Freivalds (2004), la posibilidad para que un negocio crezca y aumente sus ganancias radica en el aumento de su productividad. Este mejoramiento de la productividad se traduce en un incremento de la cantidad de producción durante las horas de trabajo empleadas, tal como se puede apreciar al final de la aplicación del paso 4 del TOC, en donde la capacidad de la producción aumentó después de la implementación de las acciones de mejora y la inversión.

Para Andrade (2011), es más sencillo medir los beneficios que la adquisición de un activo tangible (maquinaria) puede generar para la empresa, que evaluar inversiones cuando el bien adquirido se relaciona, por ejemplo, con la contratación de personal. En esta investigación, se pudo determinar el valor de la rentabilidad con mayor facilidad debido a que gran parte de esta consistió en la adquisición de un taladro y el personal contratado solo aumentó en 2 operarios.

\section{CONCLUSIONES}

Para la implementación del modelo de balance de línea, esta investigación se basó en la ejecución de los 5 pasos de la teoría de restricciones, y se obtuvieron buenos resultados al utilizar herramientas de lean manufacturing, como la detección y eliminación de desperdicios o mudas, y herramientas de mejora continua, como la metodología de las $5 S$. Dichas herramientas fueron aplicadas de manera conjunta en las acciones de mejora establecidas en el paso 2 de la teoría de restricciones.

Para implementar correctamente la metodología 5S en el área de entalle y lograr una calificación aceptable en una auditoría final, es muy importante la capacitación del encargado de producción del área, quien guiará e involucrará al personal a su cargo para cumplir con los objetivos planteados.

Los resultados obtenidos tras la aplicación del modelo de balance de línea y la demostración de la hipótesis permitieron determinar que sí hubo una mejora en la productividad, dado que al ejecutar las acciones de mejora fue posible aprovechar mejor los recursos del área y obtener una mayor capacidad de procesamiento, y, en consecuencia, disminuir los costos de producción, lo que se pudo apreciar en la estructura de costos final.

Respecto al análisis sobre la investigación realizada, en este proyecto es factible medir el valor del $\mathrm{ROI}$ de la implementación del modelo de balance de línea, según el cual se obtuvieron resultados positivos para la rentabilidad de la empresa.

Este modelo de balance de línea se podría aplicar en una primera instancia a otras organizaciones del mismo rubro, puesto que la mayoría de las empresas de procesamiento de vidrio templado del país (7 en total) suele tener la misma problemática de contar con una baja productividad en sus procesos. Esto dado que sus líneas de producción son muy similares y están conformadas por el mismo tipo de maquinaria y distribución de operarios, por lo tanto, se tendrían las mismas condiciones iniciales y se podría lograr un incremento de la productividad después de la aplicación del modelo de balance de línea, en vista de que fue beneficiosa para este caso de estudio.

\section{RECOMENDACIONES}

Se recomienda analizar la línea de procesamiento de vidrio templado de manera continua, tomando como referencia el modelo de balance de línea planteado con la finalidad de erradicar de manera oportuna las nuevas restricciones que puedan presentarse en la línea de producción y así evitar que se convierta en una limitante.

No se deben equilibrar las capacidades productivas de cada área de producción de forma individual; el objetivo debe ser obtener un flujo de producción equivalente al exigido por la demanda del área comercial.

Se pueden aplicar herramientas de lean manufacturing y de mejora continua como la detección y eliminación de mudas, y la metodología de las $5 \mathrm{~S}$, que fueron utilizadas en el modelo de balance de línea. Estas pueden ser aplicadas en otras áreas de la empresa como mantenimiento, almacenes, oficina, etc., para obtener resultados positivos.

La capacitación constante de los trabajadores (encargados de área) que apoyarán en la implementación de la tecnología $5 S$ es importante, debido a que ellos tienen contacto directo con los operarios de su turno de trabajo y son quienes manejan las máquinas. 


\section{REFERENCIAS BIBLIOGRÁFICAS}

[1] Alcalde, P. (2010). Calidad. Madrid, España: Paraninfo.

[2] Andrade, A. (2011). Análisis del ROA, ROE y ROI. Contadores y Empresas, (170). Recuperado de http://hdl.handle. net/10757/608313.

[3] Bonilla E., Díaz B., Kleeberg, F., y Noriega, M. (2010). Mejora continua de los procesos: herramientas y técnicas. Lima, Perú: Fondo Editorial de la Universidad de Lima.

[4] Calampa S. (2014). Aplicación de la Línea de Balance en el Sistema Last Planner en Proyectos de Edificaciones. (Tesis de pregrado). Pontificia Universidad Católica del Perú, Lima.

[5] Chase, R., Jacobs, F. y Aquilano, N. (2009). Administración de operaciones: Producción y cadena de suministros. México: McGraw Hill.

[6] Cuatrecasas L. y Torrell F. (2010). TPM en un entorno de Lean Management: Estrategia competitiva. España: Editorial Profit.

[7] Doberssan, J. (2010). Las 5S, Herramientas del cambio. Argentina: Editorial Universitaria de la Universidad Tecnológica Nacional.

[8] García, J., Alarcón, F. y Albarracín, J. (2004). Problemas resueltos de diseño de sistemas productivos y logísticos. Valencia, España: Universidad Politécnica de Valencia.

[9] García, R. (2006). Estudio del Trabajo: Ingeniería de métodos y medición del trabajo. México: Editorial McGraw Hill.

[10] Goldratt, E. (2004). La Meta. México: Ediciones Castillo.

[11] Hernández, J. A. (2016). Propuesta de Implementación de la herramienta de mejora continua $5 S$ en los almacenes de los talleres aeronáuticos de reparación en Bogotá D.C Colombia. Recuperado de http://hdl.handle. net/10654/15453
[12] Kikolski, M. (2017). Identification of production bottlenecks with the use of Plant Simulation software. Engineering Management in Production and Services, 8(4), 103-112.

[13] Meyers, F. (2000). Estudio de tiempos y movimientos. México: Pearson Educación.

[14] Niebel, B., y Freivalds, A. (2004). Ingeniería Industrial: métodos, estándares y diseño del trabajo. Edición. México: Alfaomega Grupo Editor S.A.

[15] OIT (1996). Introducción al estudio del Trabajo. Ginebra, Suiza. Editorial Limusa.

[16] Saidi, R., Soulhi, A., y El Alami, J. (2017). The role of the overall equipment effectiveness as a decision support tool for structuring the roadmap of a TFS transformation (Constraint theory, safety of operation, and six sigma). Journal of Theoretical and Applied Information Technology, 95(15), 3441-3449.

[17] Salazar, B. (16 de junio de 2019). Balanceo de línea. Recuperado de https://www. ingenieriaindustrialonline.com/produccion/ balanceo-de-linea/.

[18] Socconini, L. (2019). Lean Manufacturing: paso a paso. España: Editorial Marge Books.

[19] Suñé, A., Gil, F., y Arcusa, I. (2004). Manual práctico de diseño de sistemas productivos. Madrid, España: Ediciones Díaz de Santos.

[20] Rey, F. (2005). Las 5S: orden y limpieza en el puesto de trabajo. Madrid, España: FC Editorial.

[21] Vargas, H. (2004). Manual de implementación del programa 5S. Colombia: Corporación Autónoma Regional de Santander. 


\section{Production Line Balancing Model to Improve Productivity in a Tempered Glass Processing Company}

OMAR ENRique Escalante TORRES ${ }^{1}$

Received: 03/03/2021 Accepted: 25/05/2021 Published: 26/07/2021

\begin{abstract}
A line balancing model for a tempered glass processing company was developed in this study, aimed at demonstrating how its implementation helps to improve productivity. The model is based on the development of the five steps of the theory of constraints (TOC): identify the constraint, exploit the constraint, subordinate everything to the constraint, elevate the constraint, and check if there is a new constraint. Once the restriction was identified, it was exploited using improvement actions, such as the detection and elimination of mudas (wastes), and the application of the 5S methodology until a production capacity in line with demand was achieved. As economic investments had to be made, a table with a cost structure before and after the implementation of the model was prepared in order to calculate the return on investment (ROI) value to determine that the investment made promoted the increase in productivity.
\end{abstract}

Keywords: line balancing; productivity; mudas; 5S; theory of constraints.

\section{INTRODUCTION}

Most companies or organizations in the country apply various improvement theories, techniques and tools in order to increase their productivity; in many cases, this involves imitating improvement experiences that were applied in other companies and resulted in a significant increase in productivity and, consequently, in a competitive advantage over their competitors. The proper implementation of a set of continuous improvement tools is essential to achieve a real change within the organization so as to improve productivity, reduce costs and obtain a higher, sustainable profit margin over time.

The vast majority of companies in the country are SMEs, which lack fully automated production lines, and thus need to correctly balance each workstation in order to maximize the use of their resources. In general, machinery assembly and production line distribution are carried out empirically, without any improvement techniques or tools to capitalize on available resources to increase productivity. Such cases require a set of continuous improvement tools that are adjusted to the economic, spatial and demand needs of each organization.

This study was conducted in a company engaged in the processing of tempered glass used in the architectural sector as part of construction finishes. Most of the machines used for tempered glass processing are made in Italy and are often purchased from different suppliers, for economic reasons or because the supplier does not offer machinery to assemble the entire processing line. This results in different production capacities in the work areas at the time of assembly of the production line.

The purpose of this article is to implement a line balancing model that encompasses the use of continuous improvement and lean manufacturing tools for the entire production line (cutting, edging, notching and furnace areas), to address the company's problems of low productivity and wasted man-hours resulting from the failure

1 Industrial engineer from Universidad Nacional de Ingeniería. Currently working as Head of Production Planning and Control at Glasstempcorr S.A.C. (Callao, Peru).

ORCID: https://orcid.org/0000-0003-3695-5880

Corresponding author: oescalante@glasstempcorr.com.pe 
to correctly balance the production capacities of the work areas. Unlike other research and theses where the use of line balancing is developed as an improvement tool to increase productivity based on the development of strategies to balance the processing capacities of each work area, here a "line balancing model", which is a selection of continuous improvement and lean manufacturing tools that will allow achieving an increase in productivity, will be used.

To balance the tempered glass processing line, a model based on the execution of the 5 steps of the theory of constraints (identify, exploit, subordinate, elevate and repeat) will be used. Lean manufacturing tools (detection and elimination of mudas) and continuous improvement (5S methodology) will also be used to implement the improvement actions of step 2 (exploit the constraint). As mentioned by Alcalde (2010), the 5S methodology is based on order and cleanliness, as this allows the creation of a new culture within the organization. By using already existing continuous improvement tools in this research, it is possible to state that its use in the development of this line balancing model is expanding.

In order to implement the line balance model, an analysis of the work performed in all areas has to be completed; it includes the review of production reports and shift schedules to determine the time used by the operator for production under normal conditions, at a speed and work rate that should be in accordance with the demand. From this information, the production capacity of each work area under normal conditions is obtained, thus determining which has the lowest capacity and which is the limiting factor in the production line.

In view of the above, the main objective of this research is to determine whether there is a relationship between the implementation of this line balance model and an improvement or increase in productivity in the tempered glass processing company under analysis. The formulation of a hypothesis will be necessary and will subsequently be contrasted against the results to verify if there is a relationship between the model and the increase in productivity. The hypothesis is as follows: "The implementation of a line balancing model will improve the productivity of a tempered glass processing company".

A cost structure will also be prepared at the start and end of the implementation of the model to analyze the economic impact of the finished product selling price on the company and, finally, the return investment value (ROI) will be calculated to determine if the investment made was beneficial to the company.

\section{Line Balancing}

To Salazar (2019), line balancing is a method of great importance to control production, because if a correct balance of the production line is achieved, it will be possible to optimize other variables that influence productivity, including: inventory of parts to be processed, average processing time and partial dispatches of finished parts.

Suñé et al. (2004) state that when designing the production line, the tasks must be distributed so that resources are used throughout the process. The most complicated part of balancing the production line is to divide the process into workstations where a group of tasks will be executed, so that the optimal workload is achieved within the production cycle time. Regarding the latter, García et al. (2004) indicate that the objective of balancing a production line is that each workstation produces the same amount of finished material and has the same production cycle time, in order to prevent the accumulation of load to be processed.

According to Suñé et al. (2004), the steps to initiate line balancing are as follows:

1. Determine and identify all the tasks that make up the production process.

2. Determine the standard time necessary for each task.

3. Determine the necessary resources for each task.

\section{Determine the execution sequence.}

Meyers (2000) maintains that bottlenecks can be identified after implementing line balancing. As a result of the variability of demand and the use of machinery of varying capacities, different processing times are required, thus generating dynamic bottlenecks.

A balanced production line requires a correct theoretical application, redistribution of resources and even financial investments.

According to Calampa (2014), balancing the production line helps to:

- Reduce and standardize costs.

- Develop a bonus structure for achieved productivity level.

- Obtain the expected output quantity within the established deadline. 
- Improve production management.

- Increase overall productivity and staff motivation.

- Determine times and motions to improve workload distribution.

- Eliminate work-in-process inventories and bottlenecks.

- Improve process flow between production areas.

- Engage workers in the elimination of the 7 wastes or mudas of lean manufacturing (overproduction, waiting times, transportation, processes, inventories, movements and defective products).

\section{Theory of Constraints (TOC)}

The term "bottleneck" is found in line balancing. In Goldratt's (2004) Theory of Constraints (TOC), the concept of "bottleneck" is referred to as the lack of capacity of a workstation to satisfy the demand of a preceding station, thus creating a "constraint". According to Saidi et al. (2017), these "bottlenecks" determine production capacity. Consequently, the workstation with the lowest capacity among all is generating a negative impact on the total cycle time. For Kikolski (2016), this sets the pace of the production process.

Chase et al. (2009) state that the theory of constraints consists of a set of tools for continuous improvement, which is achieved by applying these 5 steps:

1. Identify the constraint. This is considered a complicated step, because the entire process must be evaluated in order to detect the activity that restricts the process flow rate. This refers not only to the process, but also to the entire company.

2. Exploit the constraint. This involves finding methods capable of increasing the capacity of the constraining activity, i.e. increasing the throughput (speed of the system to increase its profits).

3. Subordinate everything to the constraint. The constraint should operate at $10 \%$ of its capacity, since it sets the work pace.

4. Elevate the constraint. The capacity of the constraint must be increased; the use of financial resources can be considered here.
5. Check if there is a new constraint. If the initial constraint is eliminated, proceed to repeat the previous steps with the next constraint of the production process.

\section{S Methodology}

According to Hernández (2016), the purpose of the $5 S$ is to improve and maintain order in the work areas; thus, improving working conditions, organizational climate and work performance, which will result in increased productivity and improved product quality.

1. Seiri (Sort). According to Bonilla et al. (2010), it consists of classifying items into useful and non-useful, keep only those that are useful and remove those that are not because they take up space.

2. Seiton (Set in order). According to Bonilla et al. (2010), it consists of organizing the sorted items from the previous step, so that they can be easily located, thus saving time and avoiding unnecessary movements.

3. Seiso (Shine). It consists of cleaning and removing any trash; as mentioned by Rey (2005), the work area should be cleaned to keep it free of dust and encourage the worker to do it daily at the beginning and end of the workday.

4. Seiketsu (Standardize). It is based on the first three S's, and its purpose is to avoid delays in the previous stages, and its execution should be part of the daily routine. According to $\mathrm{Cu}$ atrecasas and Torrel (2010), standardization involves instilling a habit in workers to comply with the previous steps. A good organization structure and order will be essential to achieve standardization.

5. Shitsuke (Sustain). According to Dorbessan (2010), it is the most important step because it is responsible for the compliance of the previous $4 \mathrm{~S}$ 's. It proactively encourages discipline, as the benefits will be beneficial for both the company and the workers. As long as workers are self-disciplined and committed, the company will achieve great results in terms of quality and productivity levels.

\section{Mudas: Wastes}

A basic objective of lean manufacturing is to eliminate or reduce the waste or mudas produced by various tasks that do not generate any value. 
Socconini (2019) explains that lean manufacturing classifies them into seven groups:

- Overproduction: involves producing more than required.

- Waiting: time wasted when the operator is waiting for the machine to finish the cycle.

- Transportation: useless displacement of people or parts in process that does not generate value.

- Overprocessing: processes that do not increase the value of the final product.

- Inventory: materials, un-finished or finished products that exceed the demand of the commercial area.

- Motion: movements of materials or tools within the process that do not generate value and, on the contrary, increase costs.

- Defects: loss of resources that were used at the time of producing a non-conforming product.

\section{Productivity}

For García (2006), productivity is the efficiency level with which the available resources are used to achieve the objectives; he further states that the productivity of a company is examined in order to determine the problems that may affect it and, subsequently, to establish the strategies to solve them.

The ILO (1996) defines productivity as a ratio between the output produced and the input used; it adds that productivity can be affected by different external factors, as well as by deficiencies in its internal activities.

\section{METHODOLOGY}

This is an applied research with an experimental design, as the independent variable "line balancing model" is intentionally manipulated, which produces an effect on the dependent variable "productivity".

\section{Tempered Glass Production Line}

The study was conducted at Glasstempcorr, a company located in the district of Ventanilla. The plant is on a site of approximately $5000 \mathrm{~m}^{2}$ and the production line is composed of the cutting, edging, notching, washing and furnace areas. At present, its productivity is not adequate due to poor control of work between areas, reprocessing and waste. For this reason, the implementation of a line balance model based on the 5 steps of the theory of constraints is used as a continuous improvement tool, so that, at the time of implementing the improvement actions, the detection and elimination of mudas are used in conjunction with $5 \mathrm{~S}$ methodology in the work area where the restriction is detected. This sequence can be observed in Figure 1.

\section{Evaluation and Implementation of the Theory of Constraints (TOC)}

Step 1 is applied. As the historical production data is available, it is possible to obtain the production capacity of each area and an average of square meters of tempered glass processed in the last 3 months. Based on these data, it is possible to determine the area that has the lowest production capacity and, therefore, generates the constraint. In Figure 2, it is shown that the restriction of the line lies in the notching area. At this initial stage, a cost structure can be developed to calculate the unit cost value and compare it with the one obtained after the implementation of the line balancing model.

In step 2, the constraint must be exploited, therefore, actions must be taken to increase the production capacity of the notching area using only the area's own resources (operators and machinery).

\begin{tabular}{|c|c|c|c|c|c|c|c|}
\hline Start & Step 1 & \multicolumn{2}{|c|}{ Step 2} & Step 3 & Step 4 & Step 5 & Results \\
\hline \begin{tabular}{|l|} 
- Review of \\
historical \\
production \\
data (last 3 \\
months). \\
- Calculation of \\
the daily \\
production \\
capacity of \\
each work area.
\end{tabular} & \begin{tabular}{|l|} 
Identification of \\
the constraint. \\
- Using the \\
above \\
information, \\
determine \\
which \\
processing area \\
has the \\
constraint.
\end{tabular} & \begin{tabular}{|r|}
$\begin{array}{r}\text { Exploit t } \\
\text { - Prioritizin } \\
\text { company's o } \\
\text { two continu } \\
\text { tools }\end{array}$ \\
$\begin{array}{l}\text { Implemen } \\
\text { tation of } \\
\text { the } 5 \mathrm{~s}\end{array}$
\end{tabular} & $\begin{array}{l}\text { constraint. } \\
\text { he use of the } \\
\text { resources, } \\
\text { s improvement } \\
\text { applied. } \\
\text { petection and } \\
\text { elimination } \\
\text { of mudas. }\end{array}$ & \begin{tabular}{|l|} 
Subodinate \\
resources to \\
the constraint. \\
- The process \\
that has the \\
restriction is the \\
one that sets \\
the pace of \\
work.
\end{tabular} & $\begin{array}{l}\text { Elevate the } \\
\text { constraint. } \\
\text { - An economic } \\
\text { investment can } \\
\text { be made if } \\
\text { necessary. }\end{array}$ & $\begin{array}{l}\text { Check if there } \\
\text { is a new } \\
\text { constraint. } \\
\text { - If the initial } \\
\text { constraint is } \\
\text { removed, } \\
\text { the previous } \\
\text { steps are } \\
\text { repeated. }\end{array}$ & $\begin{array}{l}\text { Hypothesis } \\
\text { Testing: } \\
\text { hypothesis is } \\
\text { contrasted. } \\
\text { Economic } \\
\text { Analysis: unit } \\
\text { costs (initial - } \\
\text { final) are } \\
\text { compared. }\end{array}$ \\
\hline
\end{tabular}

Figure 1. Sequence of the line balancing model.

Source: Prepared by the author. 
Two continuous improvement tools, which are part of the proposed model, are applied in parallel; the first was the implementation of the 5S. Prior to implementation, a baseline was determined, abnormal conditions were photographed and a score was assigned to each category (sorting, order, cleanliness, standardization and maintenance) to determine the issues to be corrected. The Quality Manager, with the support of the area managers, directed the implementation, which consisted of three phases: in the first phase, the personnel involved were trained and informed; in the second phase, the methodology was implemented; and in the third phase, a follow-up of the previous implementation was conducted.

The second tool is the identification and elimination of the mudas that exist within the production process, which is used in lean manufacturing. When analyzing the notching process, mudas related to waiting times caused by lack of information on the work to be performed and stops due to unscheduled maintenance were found; a considerable percentage of defective products is also detected, resulting in waste of raw material and man-hours. Action plans must be developed to reduce this waste, such as preparing in advance all the information on the work to be performed, improving the monthly preventive maintenance program, and training personnel to reduce defective products due to mishandling. Once the improvement actions have been implemented, the production line will be balanced, as shown in Figure 3.

Step 3 consists of subordinating everything else to the current capacity of the constraint, in other words, the notching area sets the work pace and,

\begin{tabular}{|c|c|c|c|c|c|}
\hline INITIAL & $\begin{array}{l}\text { Step } 1 \\
\text { Cutting }\end{array}$ & $\begin{array}{c}\text { Step } 2 \\
\text { Edging }\end{array}$ & $\begin{array}{c}\text { Step } 3 \\
\text { Notchin } \\
\text { g }\end{array}$ & $\begin{array}{l}\text { Step } 4 \\
\text { Washing }\end{array}$ & $\begin{array}{c}\text { Step } 5 \\
\text { Temperin } \\
\mathrm{g}\end{array}$ \\
\hline MACHINERY & $\begin{array}{c}\text { Cutting } \\
\text { Machine }\end{array}$ & $\begin{array}{c}\text { Edging } \\
\text { Machine }\end{array}$ & Drill & $\begin{array}{l}\text { Washing } \\
\text { Machine }\end{array}$ & $\begin{array}{c}\text { Tempering } \\
\text { Furnace } \\
\end{array}$ \\
\hline $\begin{array}{c}\text { DAILY } \\
\text { PRODUCTION } \\
\text { CAPACITY }\left(\mathrm{m}^{2}\right)\end{array}$ & 1450 & 1200 & 800 & 1500 & 1250 \\
\hline
\end{tabular}

Figure 2. Initial production capacities.

Source: Prepared by the author.

\begin{tabular}{|c|c|c|c|c|c|}
\hline INITIAL & $\begin{array}{l}\text { Step } 1 \\
\text { Cutting }\end{array}$ & $\begin{array}{c}\text { Step } 2 \\
\text { Edging }\end{array}$ & $\begin{array}{c}\text { Step } 3 \\
\text { Notchin } \\
\text { g }\end{array}$ & $\begin{array}{l}\text { Step } 4 \\
\text { Washing }\end{array}$ & $\begin{array}{c}\text { Step } 5 \\
\text { Temperin } \\
\text { g }\end{array}$ \\
\hline MACHINERY & $\begin{array}{c}\text { Cutting } \\
\text { Machine }\end{array}$ & $\begin{array}{c}\text { Edging } \\
\text { Machine }\end{array}$ & Drill & $\begin{array}{l}\text { Washing } \\
\text { Machine }\end{array}$ & $\begin{array}{c}\text { Tempering } \\
\text { Furnace }\end{array}$ \\
\hline $\begin{array}{c}\text { DAILY } \\
\text { PRODUCTION } \\
\text { CAPACITY }\left(\mathrm{m}^{2}\right)\end{array}$ & 1450 & 1200 & 800 & 1500 & 1250 \\
\hline MACHINERY & $\begin{array}{c}\text { Cutting } \\
\text { Machine }\end{array}$ & $\begin{array}{c}\text { Edging } \\
\text { Machine }\end{array}$ & Drill & $\begin{array}{l}\text { Washing } \\
\text { Machine }\end{array}$ & $\begin{array}{c}\text { Tempering } \\
\text { Furnace }\end{array}$ \\
\hline $\begin{array}{c}\text { DAILY } \\
\text { PRODUCTION } \\
\text { CAPACITY }\left(\mathrm{m}^{2}\right)\end{array}$ & 1450 & 1200 & 1000 & 1500 & 1250 \\
\hline
\end{tabular}

Figure 3. Production capacity values after implementation of improvement actions from step 2.

Source: Prepared by the author. 
consequently, the resources used in all other production areas (operator and machinery) must be rationed to achieve a capacity that slightly exceeds that of the notching area so as to achieve a homogeneous processing capacity throughout the line. Often managers must be convinced of the need to reduce the work pace in other areas that generate excess production; therefore, it is important to have the support of management for this step.

Step 4 consists of elevating the restriction, for which an economic investment will be needed to acquire a hand drill and hire 2 operators, which will increase the capacity of the notching area to $1300 \mathrm{~m}^{2}$, as shown in Figure 4.

Step 5 consists of checking whether the limiting process has been eliminated. In this case, it can be seen that the notching area is no longer a constraint but the edging area is; therefore, the previous steps need to repeated until obtaining a production capacity in accordance with the demand posed by the commercial area.

\section{RESULTS}

The line balancing model allows comparing the production capacity before and after its implementation. In this research, a contrast of the hypothesis will be carried out first, which consists of determining if the productivity of the tempered glass production line was increased. For this purpose, information on the production from January 2019 to February 2020 is presented in Table 1, detailing the productivity rate during the months in which the line balance model was applied. The productivity rate before the implementation of the line balance model can be seen in the data presented for 2019. From January to June 2020 , an increase in the productivity rate following the implementation of the first improvement actions using the area's own resources is observed. It is important to bear in mind that in March the plant only operated until the 16th and no production was reported in April as the plant was closed due to the COVID-19 pandemic. Finally, other productivity rate values were obtained between July 2020 and February 2021 after the application of step 4 of the Theory of Constraints (TOC).

\section{Hypothesis Testing}

To test the hypothesis, productivity data is extracted from Table 1 and run in Minitab 18 to determine whether or not the data has a normal distribution.

The following graphs of the productivity comparison at the beginning and after the implementation of the model are extracted from Minitab 18.

It is determined from the normality test that the distribution is normal because the $p$-value is greater than 0.05 , the curve is bell-shaped and, if a vertical line is drawn, it intersects the mean and median.

Upon determining that, for the information collected, the data has a parametric behavior, the Student's t-test for two samples is used to compare the productivity at the beginning and at the end of the implementation of the model and to demonstrate the hypothesis.

Two-Sample T-Test and $\mathrm{Cl}$ : Initial Stage and Final Improvement

\author{
Method \\ $\mu_{1}$ : mean of Initial Stage \\ $\mu_{2}$ : mean of Final Improvement \\ Difference: $\mu_{1}-\mu_{2}$
}

Equal variances are not assumed for this analysis.

\begin{tabular}{|c|c|c|c|c|c|}
\hline FINAL & $\begin{array}{l}\text { Step } 1 \\
\text { Cutting }\end{array}$ & $\begin{array}{c}\text { Step } 2 \\
\text { Edging }\end{array}$ & $\begin{array}{c}\text { Step } 3 \\
\text { Notchin } \\
\text { g }\end{array}$ & $\begin{array}{c}\text { Step } 4 \\
\text { Washing }\end{array}$ & $\begin{array}{c}\text { Step } 5 \\
\text { Temperin } \\
\text { g }\end{array}$ \\
\hline MACHINERY & $\begin{array}{l}\text { Cutting } \\
\text { Machine }\end{array}$ & $\begin{array}{l}\text { Edging } \\
\text { Machine }\end{array}$ & Drill & $\begin{array}{l}\text { Washing } \\
\text { Machine }\end{array}$ & $\begin{array}{c}\text { Tempering } \\
\text { Furnace }\end{array}$ \\
\hline $\begin{array}{c}\text { DAILY } \\
\text { PRODUCTION } \\
\text { CAPACITY }\left(\mathrm{m}^{2}\right)\end{array}$ & 1450 & 1200 & 1300 & 1500 & 1250 \\
\hline
\end{tabular}

Figure 4. Production capacity values at the end of step 4. Source: Prepared by the author. 
Table 1. Initial Cost Structure for Step 1.

\begin{tabular}{|c|c|c|c|c|}
\hline Year & Month & Finished product $\left(\mathrm{m}^{2}\right)$ & Hours used & Productivity rate (\%) \\
\hline \multirow{12}{*}{2019} & January & 765 & 7.01 & 0.84 \\
\hline & February & 775 & 7.05 & 0.85 \\
\hline & March & 785 & 7.15 & 0.88 \\
\hline & April & 776 & 6.98 & 0.85 \\
\hline & May & 762 & 6.85 & 0.82 \\
\hline & June & 789 & 6.89 & 0.85 \\
\hline & July & 782 & 7.08 & 0.87 \\
\hline & August & 780 & 7.11 & 0.87 \\
\hline & September & 792 & 7.07 & 0.87 \\
\hline & October & 791 & 7.18 & 0.89 \\
\hline & November & 788 & 7.28 & 0.90 \\
\hline & December & 793 & 7.25 & 0.90 \\
\hline \multirow{12}{*}{2020} & January & 945 & 7.39 & 0.87 \\
\hline & February & 978 & 7.55 & 0.92 \\
\hline & March & 905 & 7.45 & 0.84 \\
\hline & April & -- & -- & -- \\
\hline & May & 931 & 7.58 & 0.88 \\
\hline & June & 945 & 7.55 & 0.89 \\
\hline & July & 1078 & 7.75 & 0.87 \\
\hline & August & 1088 & 7.85 & 0.89 \\
\hline & September & 1058 & 7.81 & 0.86 \\
\hline & October & 1138 & 7.85 & 0.93 \\
\hline & November & 1105 & 7.78 & 0.90 \\
\hline & December & 1158 & 7.89 & 0.95 \\
\hline \multirow{2}{*}{2021} & January & 1098 & 7.9 & 0.90 \\
\hline & February & 1115 & 7.68 & 0.89 \\
\hline
\end{tabular}

Source: Prepared by the author.

\section{Descriptive Statistics}

\begin{tabular}{lllll} 
Sample & N & Mean & StDev & SE Mean \\
\hline Initial Stage & 12 & 0.8640 & 0.0247 & 0.0071 \\
Final Improvement & 8 & 0.8993 & 0.0298 & 0.011
\end{tabular}

\section{Estimation for Difference}

\begin{tabular}{ll} 
Difference & $\mathbf{9 5 \% ~ C l}$ for Difference \\
\hline-0.0352 & -0.0126
\end{tabular}

\section{Test}

$$
\begin{array}{ll}
\text { Null hypothesis } & \mathrm{H}_{0}: \mu_{1}-\mu_{2}=0 \\
\text { Alternative hypothesis } & \mathrm{H}_{1}: \mu_{1}-\mu_{2}<0
\end{array}
$$

If a $p$-value $\geq 0.05$ is obtained, $\mathrm{H}_{0}$ will be accepted as a valid answer; however, if a $p$-value $<0.05$ is obtained, $\mathrm{H}_{\mathrm{a}}$ will be accepted and $\mathrm{H}_{0}$ will be rejected.

As the $p$-value is less than 0.05 , the null hypothesis is rejected and the alternative hypothesis is accepted, which indicates that the difference $\mu 1-\mu 2<0$, thus demonstrating that the productivity percentage increased as of July 2020. Therefore, this comparison proves that the hypothesis stating that "The implementation of a line balancing model will improve the productivity of a tempered glass processing company" is correct.

\section{Economic Analysis}

A comparative analysis of the average unit costs at the beginning and after the implementation of the 5 


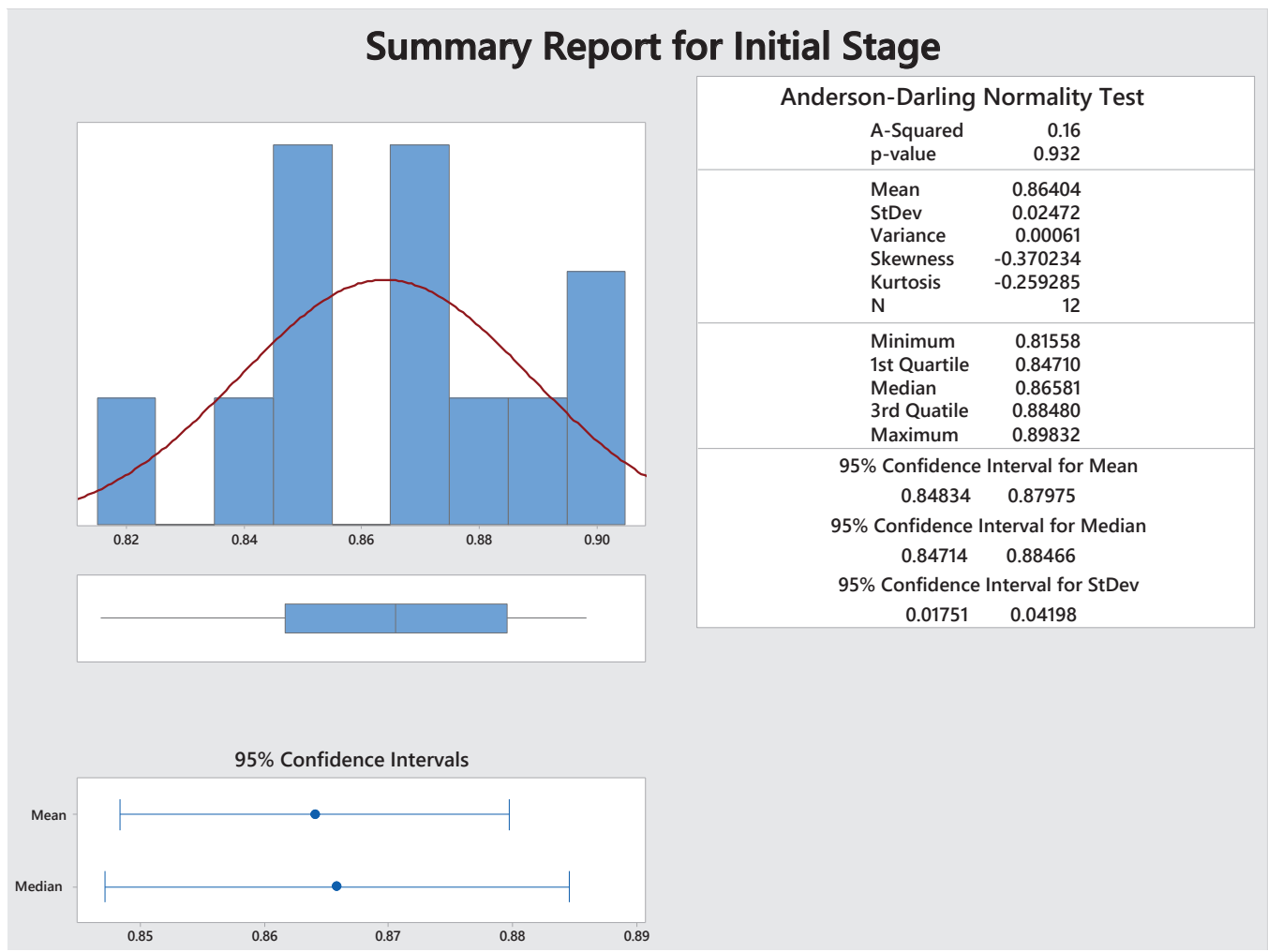

Figure 5. Graph of the normality distribution of the productivity values at the beginning of the implementation.

Source: Prepared by the author.

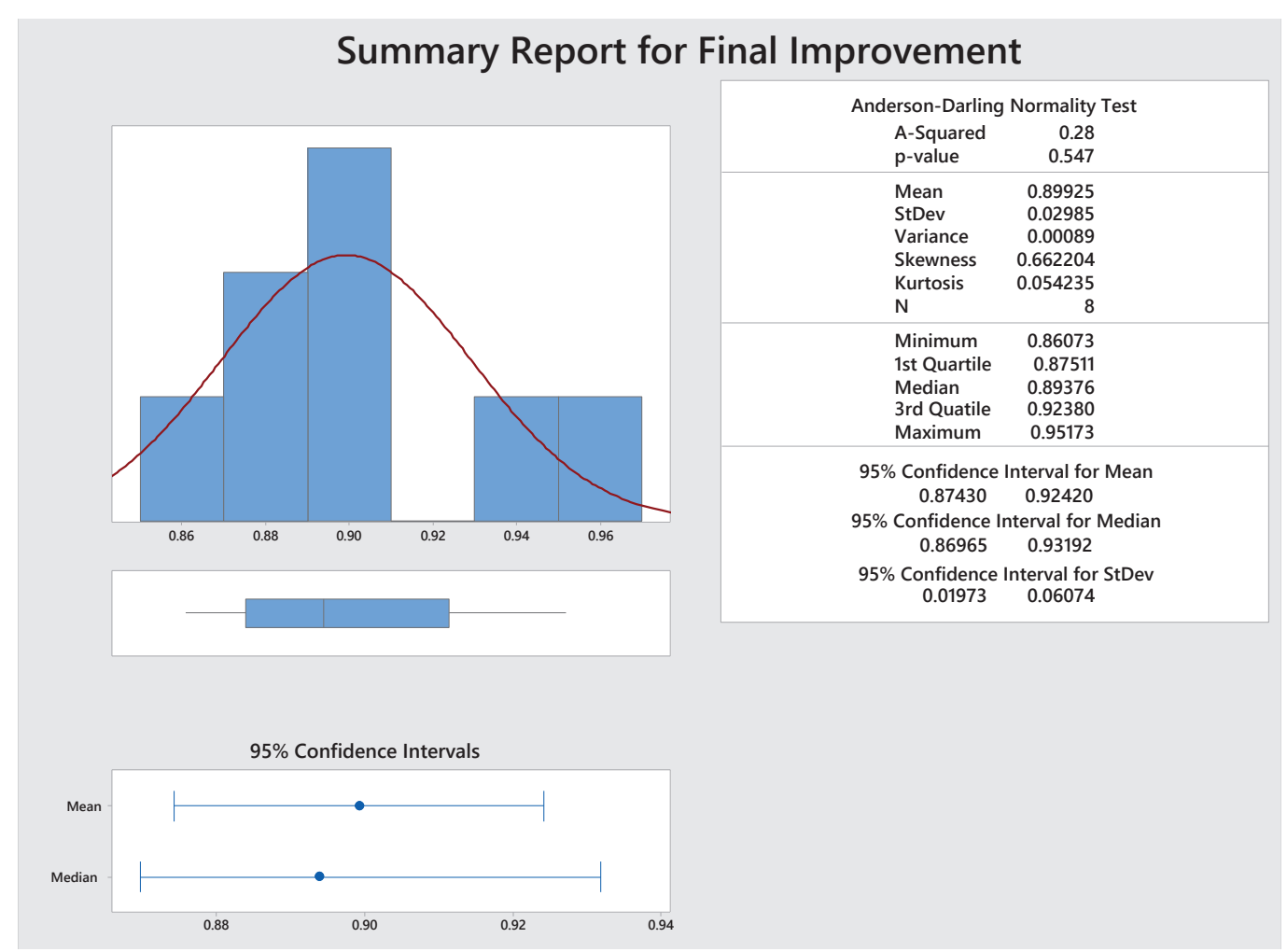

Figure 6. Graph of the normality distribution of productivity values after model implementation. Source: Prepared by the author. 
steps of the TOC will be conducted. Table 2 shows the cost structure at the beginning of the study.

Based on the table above, unit selling price per square meter is $\mathrm{S} / 77.34$ or $\$ 21.48 \approx \$ 22.00$, which is the current market price per square meter for an 8 $\mathrm{mm}$ thick glass, this calculated for a line production capacity of $800 \mathrm{~m} 2$. Table 3 shows the resulting cost structure after the application of step 4 .

Based on Table 3, unit selling price per square meter has decreased to $S / 69.16$ or $\$ 19.21 \approx \$ 20.00$ for an $8 \mathrm{~mm}$ thick glass, this calculated for a line production capacity of $1200 \mathrm{~m} 2$. The cost of the acquired machinery ( 1 drill) has been included in this table and its value has been distributed for a 5-year service life. The amount of the final selling price is below the market price, allowing the company to offer the customer a price below the market price without affecting profit.

A return on investment analysis is carried out in the notching area to determine how profitable it was for the company. From the values obtained in Tables 1 and 2, Table 4 is prepared to determine the ROI value using the formula shown below.

Table 2. Initial Cost Structure for Step 1.

Cost Structure - Initial Conditions

Average values for a demand of $800 \mathrm{~m}^{2}$

\begin{tabular}{|c|c|c|c|c|}
\hline Tempered Glass & Total Cost & Finished product $\left(\mathrm{m}^{2}\right)$ & \multicolumn{2}{|c|}{ Unit Cost } \\
\hline Material (raw glass) & S/ $\quad 39000.00$ & 800 & $\mathrm{~S} /$ & 48.75 \\
\hline MP (60 operators) & S/ $\quad 3000.00$ & 800 & $S /$ & 3.75 \\
\hline IMC (2.5) & S/ $\quad 7500.00$ & 800 & $\mathrm{~S} /$ & 9.38 \\
\hline Manufacturing cost & S/ $\quad 49500.00$ & 800 & $\mathbf{S I}$ & 61.88 \\
\hline Selling price $(25 \%)$ & S/ $\quad 61875.00$ & & $\mathbf{S} /$ & 77.34 \\
\hline
\end{tabular}

$\mathrm{IMC}=$ Indirect manufacturing cost equal to 2.5 times the cost of man power (MP).

Source: Prepared by the author.

Table 3. Cost Structure After Step 4.

\section{Cost Structure - Final Conditions}

Average values for a demand of $1200 \mathrm{~m}^{2}$

A hand drill was acquired and two operators were hired.

\begin{tabular}{|c|c|c|c|c|}
\hline Tempered Glass & Total Cost & Production $\left(\mathrm{m}^{2}\right)$ & \multicolumn{2}{|c|}{ Unit Cost } \\
\hline Material (raw glass) + Machinery & S/ $\quad 55544.00$ & 1200 & $\mathrm{~S} /$ & 46.29 \\
\hline MP (62 operators) & S/ 3100.00 & 1200 & $\mathrm{~S} /$ & 2.58 \\
\hline IMC (2.5) & S/ $\quad 7750.00$ & 1200 & S/ & 6.46 \\
\hline Manufacturing cost & 66394.00 & 1200 & $\mathbf{S} /$ & 55.33 \\
\hline Selling price $(25 \%)$ & S/ $\quad 82992.00$ & & $\mathbf{s} /$ & 69.16 \\
\hline
\end{tabular}

$\mathrm{IMC}=$ Indirect manufacturing cost equal to 2.5 times the cost of man power (MP).

Source: Prepared by the author.

Table 4. Monthly Production Structure.

\begin{tabular}{|c|c|c|c|c|c|c|}
\hline & & \multicolumn{2}{|c|}{ Production Capacity $\left(\mathrm{m}^{2}\right)$} & \multirow{2}{*}{ Unit Selling Price } & \multirow{2}{*}{ Monthly Total } & \multirow{2}{*}{ Sales Increase } \\
\hline & & Daily & Monthly & & & \\
\hline 1 & Using current resources & 800 & 20000 & S/ 77.34 & S/ 1546800.00 & \multirow{2}{*}{ S/ 528000.00} \\
\hline 2 & Exploiting the constraint & 1200 & 30000 & $S / 69.16$ & S/ 2074800.00 & \\
\hline
\end{tabular}

Source: Prepared by the author. 


$$
R O I=\frac{R E T U R N-I N V E S T M E N T}{I N V E S T M E N T}
$$

$$
\begin{array}{ll}
G=S / 528000.00 & \text { Return } \\
\mathrm{I}=\mathrm{S} / 422350.00 & \text { Investment }
\end{array}
$$

$\mathrm{ROI}=\quad 0.20$

It can be deduced from the result that for every sol invested in this line balancing model, S/ 0.20 profit will be obtained, which makes the project profitable.

\section{DISCUSSION}

This line balancing model is structured around the execution of the 5 steps of the theory of constraints. On this basis, a series of improvement actions were identified upon the application of step 2 , using only the own resources of the analyzed area in which the restriction was detected, and no additional economic investment. In order to carry out the improvement actions, elimination of mudas - waste detected in the notching area-, was contemplated. The $5 \mathrm{~S}$ were also implemented, with an emphasis on the first three, due to the low score obtained in the baseline report. During the implementation of the 5S methodology, the Quality Manager appointed the people in charge of the "notching" production area (one for each shift: day and night), who are in responsible for distributing tasks to 5 operators. For Vargas (2002), appointing a person in charge is important because it helps with the continuous compliance of this tool and also contributes to the integration and training of the workers.

The line balancing model (independent variable) applied influenced productivity (dependent variable), in view of the fact that the hypothesis was validated by means of statistical software. After the analysis of the final cost structure, a unit selling price of $S / 69.11$ was obtained, which is lower than the unit selling price of the initial cost structure, $S / 77.34$. This outcome benefits the company because the product can be offered at a below-market price, thus allowing it to attract more customers while maintaining its profit margin. The production line will be balanced at a capacity of $1200 \mathrm{~m}^{2}$, which represents an increase of $400 \mathrm{~m}^{2}$ over the initial capacity; this line balance allowed the company to take advantage of the area's resources: use and availability of the machinery, and time used by the operators.
Niebel and Freivalds (2004) believe that the possibility for a business to grow and increase its profits lies in increasing its productivity. Such productivity improvement translates into an output increase during the working hours employed, as evidenced at the end of the application of step 4 of TOC, where the production capacity increased after investment and implementation of improvement actions.

Andrade (2011) believes that it is easier to measure the benefits that the acquisition of a tangible asset (machinery) generates for the company, than to evaluate investments when the acquired asset is related, for example, to the hiring of personnel. In this study, it was easier to determine profitability because a large part of the investment consisted of the acquisition of a drill and hiring only 2 additional workers.

\section{CONCLUSIONS}

To implement the line balancing model, this study relied on the execution of the 5 steps of the theory of constraints; good results were obtained by using lean manufacturing tools, such as the detection and elimination of wastes or mudas, and continuous improvement tools, such as the $5 \mathrm{~S}$ methodology. These tools were jointly applied in the improvement actions established in step 2 of the theory of constraints.

To properly implement the 5S methodology in the notching area and achieve an acceptable score in a final audit, it is very important to train the area's production manager, who will guide and engage the personnel in charge of the area to meet the objectives set.

Based on the results obtained after the implementation of the line balancing model and the demonstration of the hypothesis, productivity was indeed improved, as the improvement actions made it possible to better capitalize on the area's resources and obtain a greater production capacity and, consequently, lower production costs, as can be observed in the final cost structure.

As for the analysis of this study, ROI value of the implementation of the line balancing model can be measured, according to which positive results were obtained in terms of profitability.

This line balancing model could be applied to other organizations within the same industry, as most tempered glass manufacturing companies in the country ( 7 in total) tend to have low productivity in their production processes. This happens because 
their production lines are very similar and have the same type of machinery and distribution of operators; therefore, they would have the same initial conditions and an increase in productivity could be achieved after the application of the line balancing model, considering that it was beneficial for this case study.

\section{RECOMMENDATIONS}

The tempered glass production line should be analyzed continuously, taking as a reference the line balancing model proposed to timely eradicate new constraints that may arise in the production line and thus prevent it from becoming a limitation.

Production capacities of each production area should not be balanced individually; the goal should be to obtain a production flow equivalent to that required by the demand of the commercial area.

Lean manufacturing and continuous improvement tools, such as the detection and elimination of $\mathrm{mu}$ das and the $5 S$ methodology, which were used in the line balancing model, can be applied. All these tools can also be applied in other areas of the company, such as maintenance, warehouse, office, etc., to obtain positive results.

Constant training of the workers (area managers) who will support the implementation of $5 \mathrm{~S}$ technology is important, as they maintain direct contact with the operators assigned to their work shift and are the ones who operate the machines.

\section{REFERENCES}

[1] Alcalde, P. (2010). Calidad. Madrid, Spain: Paraninfo.

[2] Andrade, A. (2011). Análisis del ROA, ROE y ROI. Contadores y Empresas, (170). Retrieved from http://hdl.handle.net/10757/608313.

[3] Bonilla E., Díaz B., Kleeberg, F., \& Noriega, M. (2010). Mejora continua de los procesos: herramientas y técnicas. Lima, Peru: Fondo Editorial de la Universidad de Lima.

[4] Calampa S. (2014). Aplicación de la Línea de Balance en el Sistema Last Planner en Proyectos de Edificaciones. (Undergraduate thesis). Pontificia Universidad Católica del Perú, Lima.

[5] Chase, R., Jacobs, F., \& Aquilano, N. (2009). Administración de operaciones: Producción y cadena de suministros. Mexico: McGraw Hill.
[6] Cuatrecasas L., \& Torrell F. (2010). TPM en un entorno de Lean Management: Estrategia competitiva. Spain: Editorial Profit.

[7] Doberssan, J. (2010). Las 5S, Herramientas del cambio. Argentina: Editorial Universitaria de la Universidad Tecnológica Nacional.

[8] García, J., Alarcón, F., \& Albarracín, J. (2004). Problemas resueltos de diseño de sistemas productivos y logísticos. Valencia, Spain: Universidad Politécnica de Valencia.

[9] García, R. (2006). Estudio del Trabajo: Ingeniería de métodos y medición del trabajo. Mexico: Editorial McGraw Hill.

[10] Goldratt, E. (2004). La Meta. Mexico: Ediciones Castillo.

[11] Hernández, J. A. (2016). Propuesta de Implementación de la herramienta de mejora continua $5 S$ en los almacenes de los talleres aeronáuticos de reparación en Bogotá D.C Colombia. Retrieved from http://hdl.handle. net/10654/15453

[12] Kikolski, M. (2017). Identification of production bottlenecks with the use of Plant Simulation software. Engineering Management in Production and Services, 8(4), 103-112.

[13] Meyers, F. (2000). Estudio de tiempos y movimientos. Mexico: Pearson Educación.

[14] Niebel, B., \& Freivalds, A. (2004). Ingeniería Industrial: métodos, estándares y diseño del trabajo. Edición. México: Alfaomega Grupo Editor S.A.

[15] OIT (1996). Introducción al estudio del Trabajo. Ginebra, Switzerland. Editorial Limusa.

[16] Saidi, R., Soulhi, A., \& El Alami, J. (2017). The role of the overall equipment effectiveness as a decision support tool for structuring the roadmap of a TFS transformation (Constraint theory, safety of operation, and six sigma). Journal of Theoretical and Applied Information Technology, 95(15), 3441-3449.

[17] Salazar, B. (June 16, 2019). Balanceo de línea. Retrieved from https://www. ingenieriaindustrialonline.com/produccion/ balanceo-de-linea/.

[18] Socconini, L. (2019). Lean Manufacturing: paso a paso. Spain: Editorial Marge Books.

[19] Suñé, A., Gil, F., \& Arcusa, I. (2004). Manual práctico de diseño de sistemas productivos. Madrid, Spain: Ediciones Díaz de Santos. 
[20] Rey, F. (2005). Las 5S: orden y limpieza en el puesto de trabajo. Madrid, España: FC Editorial.

[21] Vargas, H. (2004). Manual de implementación del programa 5S. Colombia: Corporación Autónoma Regional de Santander. 